\title{
ATMOSPHERIC EFFECTS AND CALIBRATIONS
}

\author{
COLIN R. MASSON \\ Center for Astrophysics, 60 Garden St., Cambridge, MA 02138
}

\begin{abstract}
Atmospheric opacity severely attenuates millimeter and submillimeter signals. Fluctuations in refractive index limit the angular resolution of interferometers. Conventional phase calibration does not remove atmospheric phase fluctuations, but new techniques may.
\end{abstract}

\section{INTRODUCTION}

Observations in the millimeter ( $\mathrm{mm}$ ) and submillimeter (smm) range are strongly affected by tropospheric water and, to a lesser extent, by other atmospheric constituents, such as oxygen and ozone. The opacity of the atmosphere increases generally with frequency until observations from the ground are virtually impossible for $\nu>1 \mathrm{THz}$. As receiver technology has improved, there has been a move to higher and drier sites to minimize the atmospheric attenuation.

Path length fluctuations, which distort the incoming wavefronts and produce phase errors in interferometers, were not a problem for early, low-resolution telescopes, but have become more important with the advent of large antennas (Altenhoff et al. 1987) and interferometers (Wright \& Welch 1990).

Both opacity and phase stability vary from one site to another and from one time to another. For the best $\mathrm{mm}$ and smm interferometry, therefore, it is important to select the best possible site and to plan observations to make the best use of the seasonal and diurnal variations. Some mitigation of phase errors may be possible by special calibration of the data.

\section{OPACITY}

Observations in the smm are restricted to the semi-transparent windows between atmospheric lines. The lines are intrinsically narrow, and the opacity far from the line centers is predominantly due to pressure broadening. The theory for lineshapes in the far wings is not exact, and all models include a simple analytic form for the lineshape and add an empirical continuum term to match the observed opacity in the low frequency windows (e.g. Liebe 1989).

Since the opacity in the windows is due to pressure broadening, the opacity at any site is roughly proportional to the product of pressure and absolute humidity, integrated along the line of sight. In practice, water is concentrated in the lower atmosphere, with a 'scale height' of about $2 \mathrm{~km}$, so it is usually a good approximation to say that all the water above a given site is close to the altitude of that site. Therefore higher sites have less opacity than lower sites for the same PWV, and it is better to characterize sites by their opacity at some 
reference frequency, rather than by their PWV.

A convenient reference frequency for comparison is $225 \mathrm{GHz}$, where an NRAO radiometer has been used extensively for site testing. According to the theoretical models (Liebe 1989) and extrapolations from experimental data (Masson 1989), the opacity at $225 \mathrm{GHz}, \tau_{225}$, is given by the formula

$$
\tau_{225} \approx 0.01+0.04 \times P W V(\mathrm{~mm})
$$

at the elevation of Mauna Kea, where the offset of 0.01 is due to oxygen. The opacities at other frequencies have been determined empirically relative to the $225 \mathrm{GHz}$ radiometer at Mauna Kea. There is a good correlation and the results are in agreement with atmospheric emission spectra (Hills et al. 1978). The opacity at $345 \mathrm{GHz}$ is given by

$$
\tau_{345} \approx 0.05+2.5 \times \tau_{225}
$$

and the opacity in the highest frequency windows is approximately

$$
\tau_{820} \approx \tau_{690} \approx \tau_{490} \approx 20 \times\left(\tau_{225}-0.01\right)
$$

Extensive opacity measurements have recently been made by NRAO (Hogg 1992) and the University of Arizona (Martin, private communication). I will not discuss the different sites in detail, but will discuss Mauna Kea, since it is very well-studied and is an important smm site. In cooperation with Smithsonian Astrophysical Observatory (SAO) and the Caltech Submillimeter Observatory, NRAO has operated a radiometer on Mauna Kea for 3 years (Hogg 1992; Masson 1992). Figure Ia shows the opacity quartiles and the minimum value plotted as a function of hour of the day, and figure $\mathrm{Ib}$ shows the same plot for month of the year. The overall median opacity is 0.097 , and the best is 0.023 , corresponding to about $0.3 \mathrm{~mm}$ PWV. Fig. Ia shows the well-known diurnal variation, where the days have higher opacity as the inversion layer rises. Similar effects have been seen at many other sites. Note, however, that the opacity can be good at all hours of the day under the best conditions. All sites have a seasonal variation also, and Mauna Kea is no exception, with the first half of the year being significantly better than the second.

Amplitude calibration for interferometer data is similar to that for singledish data, using the well-established 'chopper cal' method (e.g. Kutner \& Ulich 1981). The final stage of calibration is to account for the telescope efficiency by making observations of standard sources, such as planets.

While there are no fundamental methods to mitigate the effects of opacity other than choosing the best possible site, a significant gain in efficiency can be made by studying the seasonal and diurnal weather patterns and scheduling smm observations in the most propitious times. Further gains will be made by dynamic scheduling to adjust the observing program to the weather on a daily basis, as is planned for the SAO Submillimeter Array (SMA).

\section{PHASE}

The total excess path length through the atmosphere from sea level is about $2.3 \mathrm{~m}$, with typically $1 \sim 10 \%$ of this due to water vapor. However, the water 


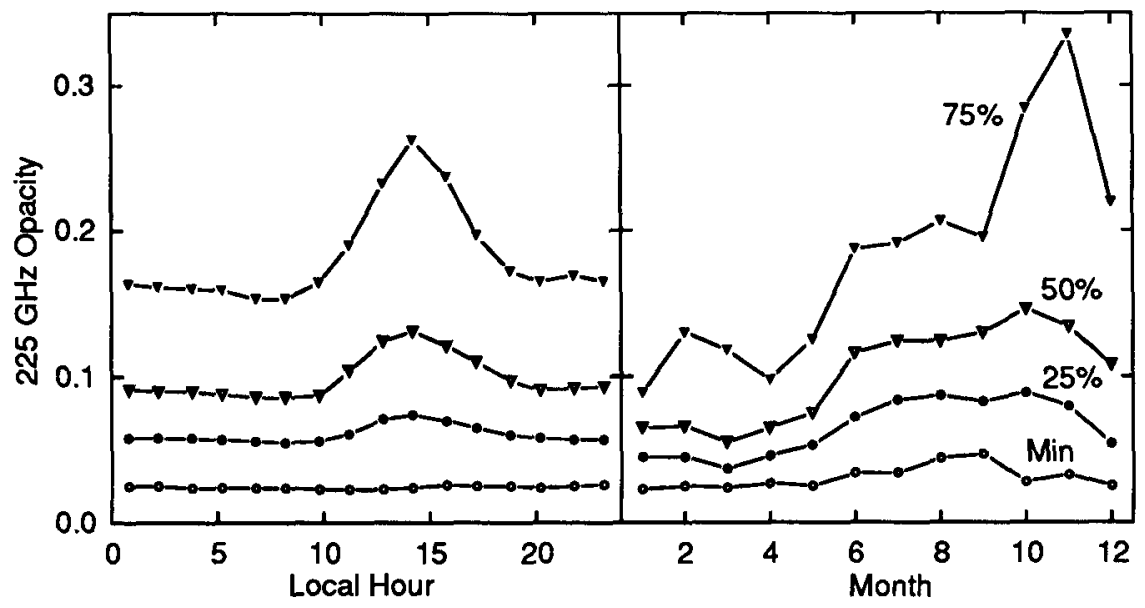

FIGURE I Opacity at Mauna Kea. a) Quartiles and minimum as as function of hour of day. b) Quartiles and minimum as as function of month.

vapor is not well mixed, since the atmospheric temperature is close to the condensation point of water, and the irregular distribution of water vapor distorts the wavefronts and limits the resolution of radio telescopes. In the radio regime, the atmosphere is nearly non-dispersive at all frequencies where it is transparent (Liebe 1989), and each $1 \mathrm{~mm}$ of PWV corresponds to a path length of $\sim 6 \mathrm{~mm}$.

The turbulent fluctuations of water vapor content are sketched in the cartoon in figure II. The larger turbulent scales have larger amplitude, so the rms phase fluctuations measured by an interferometer of baseline, $b$, increase monotonically with $b$, typically as a power law $\Delta \phi \propto b^{\beta / 2}$. This can be related to the structure function of the atmospheric turbulence (e.g. Sramek 1990). The exponent $\beta / 2$ is always less than 1 , so the average tilt of the incoming wavefronts increases as $b$ decreases. From the cartoon in figure II, it can be seen that the small scales cause independent variations at the two antennas, so that the rms phase difference between the two antennas is $\sqrt{2}$ greater than the rms path above one antenna. For atmospheric spatial scales greater than $b$, the path difference is reduced by the correlation between the two antennas. An interferometer of baseline $b$ is dominated by atmospheric scales $\approx k b$, where $k$ depends on $\beta / 2$, but is typically $\sim 5$.

The time variations of the atmospheric phase are usually approximated by a 'frozen screen' model, in which the turbulent atmosphere is supposed to be transported bodily across the telescope. This corresponds to the assumption that the internal velocities are smaller than the bulk velocity, which is probably a reasonable approximation for small scales. Figure III shows a typical frequency spectrum of phase variations measured on the SAO test interferometer at Mauna Kea. At high frequencies the spectrum is well fit by a power law of the form $\phi(f) \propto f^{-\delta}$, with a typical slope of $\delta=1.25$. There is a break in the slope at a corner frequency, $f_{c} \sim 0.01 \mathrm{~Hz}$, and the low frequency slope is 0.25 . Under the frozen screen hypothesis, the temporal frequency of the interferometer phase 


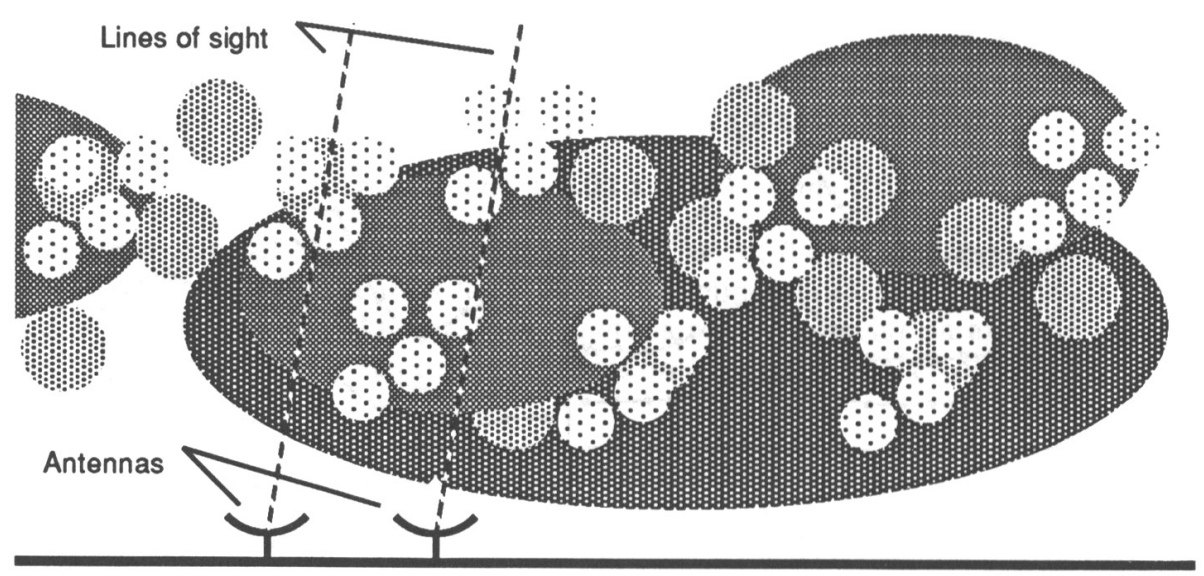

FIGURE II Cartoon showing the effect of turbulence of different scales. Shaded areas represent clouds of water vapor. The largest scales have the largest amplitudes, but the paths to the two antennas are partially correlated. The smaller scales have less amplitude, but no correlation.

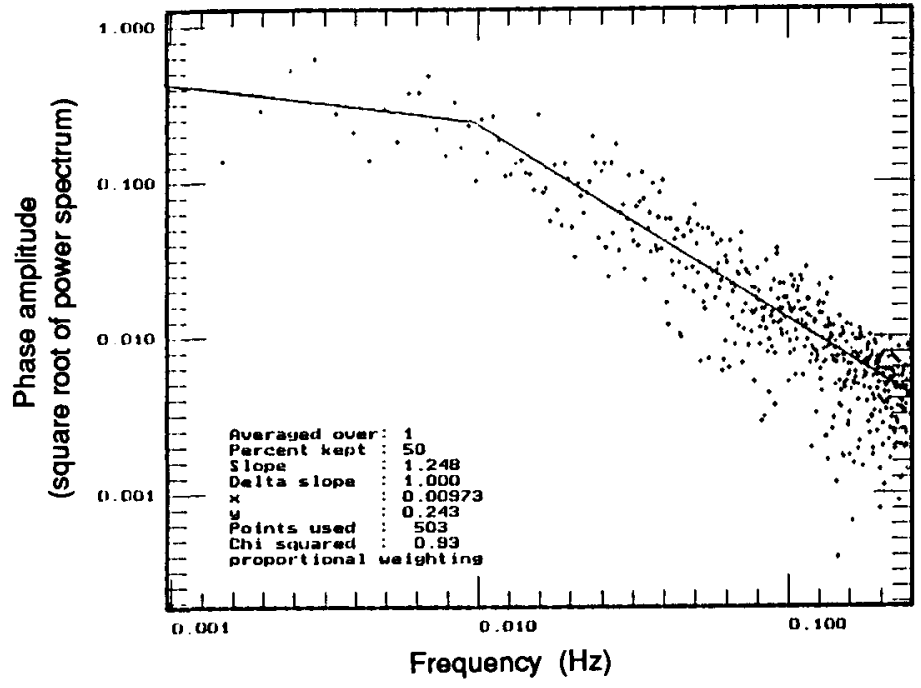

FIGURE III Typical frequency spectrum of the phase fluctuations observed on a $100 \mathrm{~m}$ baseline at Mauna Kea. The curve plotted is the square root of the power spectrum. 
variations is just proportional to the wind velocity divided by the spatial wavelength of the turbulence, and there is a direct connection between the power law exponent $\beta / 2$ which describes the relative amplitudes of the different spatial scales of turbulence in the atmosphere, and the exponent $\delta$, which describes the frequency spectrum of the phase fluctuations. It can be shown that $\delta=\beta / 2+0.5$ (Tatarskii 1971). The corner frequency is given by $f_{c} \sim k b / v$, where $v$ is the wind speed. At frequencies below the corner, the spectrum has a slope of $(\delta-1)$. The details of the shape of the corner depend on the direction of the wind relative to the baseline. The corner frequency is proportional to baseline length, but above the corner frequency the frequency spectrum is independent of baseline length, both in amplitude and slope. For example, the fast phase variations seen in VLBI should be the same as the variations seen at corresponding frequencies in connected-element interferometry.

We now have enough information to discuss phase measurements and phase calibration. Most measurements of rms path as a function of baseline use a highpass filter of some sort, often imposed by the finite length of the observation. Consideration of the spectrum in figure III shows that this lower cutoff frequency ought to be much lower than the corner frequency. If the scan length is too short, then the rms phase will be underestimated, and the underestimate will be systematically larger for longer baselines, affecting any determination of $\gamma$. The necessary time can be rather long. For a $100 \mathrm{~m}$ baseline on Mauna Kea, for example, a scan length of 15 minutes reduces the measured rms by about $5 \%$. In comparing the results of different measurements, a correction should also be made for elevation differences. For short baselines, where the turbulence is 3 -dimensional, the phase should scale as $(\operatorname{cosec}(\text { elevation }))^{1 / 2}$ and for long baselines, it should scale as cosec(elevation).

Phase measurements at many sites prior to 1989 have been discussed in the IAU Symposium of Radioastronomical Seeing (Baldwin and Wang 1990). Observations from operating observatories usually cover only a small sample of times. Recently, however, two groups, one at NRO (Ishiguro et al. 1990), and one at SAO, have used small interferometers observing geostationary satellites to make continuous measurements of atmospheric phase. The SAO survey at Mauna Kea is still in progress but some preliminary results from the first 21 months of observations are presented here.

The SAO system consists of a pair of $1.8 \mathrm{~m}$ commercial satellite dishes with HEMT amplifiers, forming an interferometer with a baseline of $100 \mathrm{~m}$ near the JCMT on Mauna Kea. The system receives a beacon tone from the GStarA2 satellite (elevation $30^{\circ}$ ) at $12 \mathrm{GHz}$. The rms phase is calculated for each 15 minute section of the data, after subtracting a mean and slope to remove the effects of satellite motion. Fig IV shows the measured rms path length, divided by $\sqrt{2}$ to correct to $90^{\circ}$ elevation, and plotted as functions of hour of day, and month. The overall median path length is 112 microns. The diurnal variation is even more pronounced for phase than opacity, and the daytime phase is degraded even under the best conditions. There is a general correlation between rms phase and opacity, but the scatter is large and all combinations of opacity and rms phase are possible.

Both $\beta / 2$ and $f_{c}$ cover a wide range. For the Mauna Kea data, the typical value of $\beta / 2$ is 0.75 , less than the value of $5 / 6$ expected for Kolmogorov turbulence, but there is a wide variation, with values from $0.2-1$. At the longer 


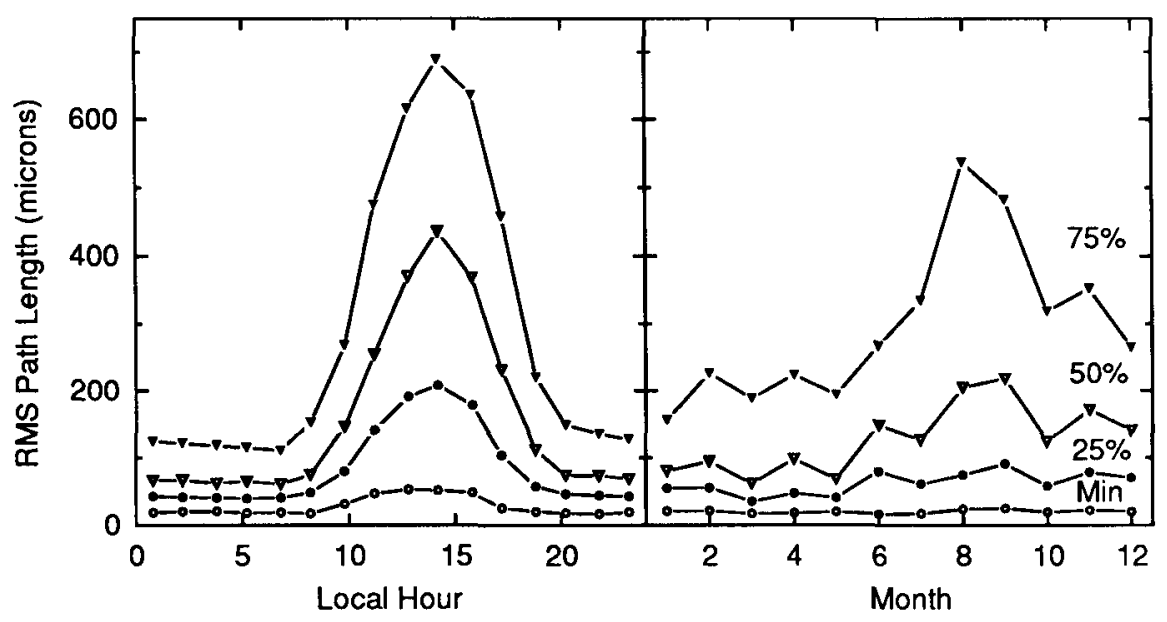

FIGURE IV Diurnal and seasonal variation of interferometer phase on Mauna Kea. The data are for a baseline of $100 \mathrm{~m}$ and have been corrected to the zenith.

baselines of the VLA, the typical value of $\beta / 2$ is $\sim 0.4$ (Sramek 1990). The corner frequency at the $100 \mathrm{~m}$ baseline on Mauna Kea ranges from $0-0.06 \mathrm{~Hz}$.

These data can be used to calculate the limiting angular resolution by using the spectral information to estimate $\beta / 2$, and hence extrapolating the rms path to baselines longer than $100 \mathrm{~m}$. For each hour of data, we have calculated the baseline, $b_{\max }$, at which the rms path corresponds to 1 radian at $345 \mathrm{GHz}$. The limiting resolution, $\theta_{\min }$, of an interferometer is then given approximately by $\theta_{\min }=0.7 \lambda / b_{\max }$. Figure $\mathrm{V}$ shows the distribution of $\theta_{\min }$. The dependence of resolution on frequency is small, with $\theta_{\min } \propto f^{(2 / \beta)-1} \sim f^{0.3}$. The median resolution is $1^{\prime \prime} 2$ at $345 \mathrm{GHz}$, corresponding to a baseline of $108 \mathrm{~m}$. During nights in the first half of the year, substantially better resolution will be obtained.

\section{PHASE CALIBRATION}

It is often supposed that the classic method of observing a calibrator every 20 minutes corrects for atmospheric phase, but this is untrue, except for the longest baselines. To correct for the atmosphere, the calibration must be done faster than the timescale of phase fluctuations. Based on the Mauna Kea data, where $f_{c}$ is typically $0.01 \mathrm{~Hz}$ for the $100 \mathrm{~m}$ baseline, the calibration interval, $\Delta t$, should satisfy the inequality $\Delta t($ seconds $)<0.25 b$ (meters). Thus calibration every 20 minutes would be inadequate for baselines shorter than $4 \mathrm{~km}$. For almost all existing interferometers, then, the standard calibration is completely ineffective in removing atmospheric phase errors. Furthermore, even if the calibrator could be observed frequently, the path through the atmosphere would differ from that to the program source, limiting the effectiveness of calibration for short baselines. These effects have been discussed in detail by Masson (1989) and Holdaway $(1992 \mathrm{a}, \mathrm{b})$.

Even though a calibration will not help for atmospheric phase, it is required 


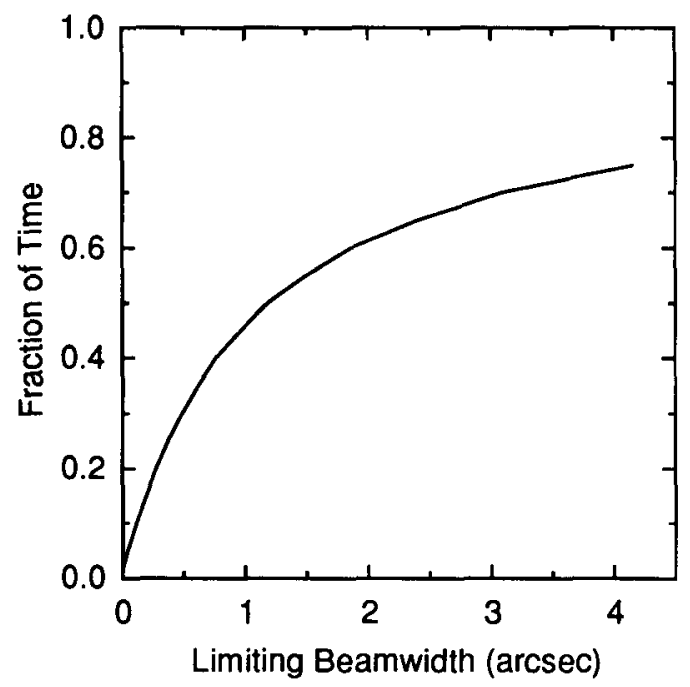

FIGURE $V$ The limiting resolution of an interferometer on Mauna Kea at $345 \mathrm{GHz}$. The values are extrapolated from measurements at $100 \mathrm{~m}$ baseline.

to compensate for the following effects: Geometry errors (e.g. baseline): This needs a close calibrator since the residual errors are proportional to distance. Instrumental phase drift, for which calibration must be sufficiently frequent to follow the drift. A strong calibrator is needed to minimize noise added by the calibration measurement itself. There is therefore a tradeoff between choosing a nearby (probably weak) and a strong (probably distant) calibrator. Given source statistics and telescope sensitivity, it is possible to estimate the typical phase error obtainable. For the SMA, operating at $230 \mathrm{GHz}$, the optimum is a 0.75 Jy calibrator, which will typically be 0.15 radians away from the program source, giving a residual error of 20 microns after calibration (Masson 1989). If self calibration is possible for long timescale phase errors, it is preferable to choose a stronger and more distant calibrator, which will add less noise but leave larger residual geometric errors.

\section{IMPROVED CALIBRATION TECHNIQUES}

At the highest frequencies, there are very few strong point sources, and the atmosphere reduces the sensitivity of interferometers. While thermal dust sources may be useful for short baselines, they have low brightness temperatures and direct calibration may not be possible at the longest baselines. One possible method for avoiding this difficulty is to observe simultaneously at a lower frequency and extrapolate the calibration to the higher frequency, as is planned for the SMA (Masson 1989).

A number of possible schemes have been proposed or tested for improving phase calibration. The best known is self-calibration, pioneered in VLBI and brought to a high standard at the VLA (Schwab 1980). This involves an iterative 
fitting of the data to a model of the source and a model of the atmospheric phase. Self calibration is most useful at longer baselines, where the timescales are longer, and requires strong sources. It will therefore be of limited use in the smm, where few sources have enough brightness temperature to give good enough signal/noise ratios on the atmospheric timescale. It may be more useful for correcting geometric errors for which the timescale is many hours, permitting the use of weaker sources.

Holdaway $(1992 \mathrm{a}, \mathrm{b})$ has proposed a number of schemes involving simultaneous observations of calibrators, or fast switching between source and calibrator to overcome the time limits for correcting atmospheric phase.

A promising technique which has been attempted several times, but which has recently been demonstrated to work in a test at BIMA (Welch 1992) is to use radiometric measurements to determine the PWV along the path of each antenna and use these measured values to deduce a phase correction. This scheme is in principle straightforward to apply at smm frequencies, where the opacity of water vapor is high. However, extremely high stability, $\Delta T / T<10^{-4}$, is required for the receivers to make use of this technique.

\section{ACKNOWLEDGMENTS}

I wish to thank many people for help and advice, especially $M$. Ishiguro and $R$. Hills, and F. Owen, who is responsible for the NRAO radiometer campaign.

\section{REFERENCES}

Altenhoff, W. J., Baars, J. W. M., Downes, D., Wink, J. E., 1987. A\&A, 184, 381.

Baldwin, J. E. \& Wang Shouguan, 1990. IAU Symp on Radio Astronomical Seeing, eds J. E. Baldwin \& Wang Shouguan. IAP, Beijing.

Liebe, H. J. 1989. Intl. J. IR \& MM Waves, 10, 631.

Hills, R. E. et al. 1978. IR Phys., 18, 819.

Hogg, D. E. 1992. Millimeter Array Memo 79. NRAO.

Holdaway, M. 1992. Millimeter Array Memo 84. NRAO.

Holdaway, M. 1992. Millimeter Array Memo 88. NRAO.

Ishiguro, M., Kanzawa, T., \& Kasuga, T. 1990. IAU Symp on Radio Astronomical Seeing, eds J. E. Baldwin \& Wang Shouguan. IAP, Beijing.

Kutner, M. L., \& Ulich, B. L. 1981. ApJ 250, 341.

Masson, C. R. 1989. SMA Technical Memo 5. SAO, Cambridge.

Masson, C. R. 1990. SMA Technical Memo 12. SAO, Cambridge.

Masson, C. R. 1992. SMA Technical Memo 60. SAO, Cambridge.

Schwab, F. R. 1980. Proc. S.P.I.E. 23118

Sramek, R. A. 1990. IAU Symp on Radio Astronomical Seeing, eds J. E. Baldwin $\&$ Wang Shouguan. IAP, Beijing.

Tatarskii, V. I. 1971. The Effects of the Turbulent Atmosphere on Wave Propagation, Israel Program for Scientific Translations, Jerusalem.

Welch, W. J. 1993. These proceedings.

Wright, M. C. H., \& Welch, W. J. 1990. IAU Symp on Radio Astronomical Seeing, eds J. E. Baldwin \& Wang Shouguan. IAP, Beijing. 


\section{DISCUSSION}

A. Rudolph What are the plans for the SMA concerning calibration of the atmospheric phase, given your pessimism about being able to track atmospheric fluctuations at $\mathrm{mm}$ and sub- $\mathrm{mm}$ wavelengths?

C. Masson As in other small interferometer, standard phase calibration will be unable to remove atmospheric fluctuations. The SMA will have an additional problem due to the low flux densities of calibrators at the highest frequencies. We will therefore make calibration measurements at $230 \mathrm{GHz}$ and extrapolate to higher frequencies.

L. Carlstrom You quoted that $\sim 30 \%$ of the time the JCMT-CSO baseline would be coherent at $345 \mathrm{GHz}$. That figure included data from all hours ( 24 hours/day), what would the fraction be if you only use night-time data?

C.Masson If you assume that the phase is very bad for $\sim 10$ hours during the day, the fraction of good time during the night might be $\sim 50 \%$.

\section{R, Ekers Comment:}

1) Although it is common practice at the VLA to make frequent observations of calibrators there in not much evidence especially for the more compact arrays that it removes the atmospheric phase errors.

2) The method of simultaneously observing a calibrator with an interleaved subset of an array was used successfully with the Westerbork array for the solar gravitational deflection experiments in the early 70 's.

\section{Masson I agree.}

